\title{
Gender-Dynamiken in der Rekonstruktion von Bildungsprozessen in Medienprojekten
}

Barbara Stauber

Dieser Beitrag ${ }^{1}$ basiert auf Erfahrungen aus einem aktuellen Praxisforschungsprojekt des tifs (Tübinger Institut für frauenpolitische Sozialforschung). Es wurde in Auftrag gegeben von der Landesstiftung Baden-Württemberg zur Evaluation ihres Programms «Jugend und verantwortungsvolle Mediennutzung», mit dem sie medienpädagogische Projekte in unterschiedlichen Jugendhilfe-Kontexten (Projekte der offenen und der verbandlichen Jugendarbeit, der Hilfen zur Erziehung, der Jugendberatung etc.) förderte. Damit kommen Zielgruppen in den Blick, die nicht immer im Zentrum der medienpädagogischen Debatten stehen. Das Projekt wurde von der Autorin gemeinsam mit Gerrit Kaschuba und Helga Huber in zwei Evaluationsphasen (2003 bis 2006) durchgeführt (vgl. Huber u. a. 2005 und 2007). Während die erste Phase das Programm im Hinblick auf «Medien und Persönlichkeitsentwicklung» evaluierte, standen in der zweiten Phase gesellschaftliche Bezüge der Medienpädagogik im Zentrum. Die beiden Phasen umfassten jeweils eine Fragebogenerhebung, auf die hier nicht weiter eingegangen wird, und jeweils sechs qualitative Fallstudien. Im Hinblick auf Letztere wurde für die beiden Phasen ein unterschiedlicher methodischer Zuschnitt gewählt. Diese Vorgehensweise erlaubt es nun, die jeweiligen Methoden-Kombinationen daraufhin zu überprüfen, inwieweit sie unter der Gender-Perspektive ertragreich waren. Nach einem kurzen Blick auf die in dieser zweiteiligen Evaluation angewandten Methoden wird danach gefragt, ob und wie es mit ihnen gelang, Bildungsprozesse in den untersuchten medienpädagogischen Projekten unter der Perspektive von Gender-Dynamiken zu erfassen. Sodann werden einige Ergebnisse im Hinblick auf das Einbeziehen von Gender-Dynamiken dargestellt - auf der medienpädagogisch-praktischen Ebene, auf der Ebene der Rekonstruktion von Bildungsprozessen sowie auf der Ebene der Forschungsinteraktion.

\section{Die in den beiden Evaluationsphasen angewandten Methoden}

Der Gegenstand der beiden Evaluationsphasen war heterogen: Die Grundgesamtheit von 128 bzw. 86 geförderten Projekten wies eine grosse Bandbreite medienpädagogischer Arbeit auf, in unterschiedlichen Einrichtungen, unter unterschiedlichen Rahmenbedingungen, mit unterschiedlichen Medien und unterschiedlichen medienpädagogischen Zielsetzungen. Auch das Spektrum der in den Projekten vertretenen Fachlichkeit war sehr breit: Es reichte von Professionellen der Ju-

Die grundlegende Struktur und zentrale Gedanken dieses Beitrags wurden zusammen mit Gerrit Kaschuba entwickelt. 
gendhilfe, die über Medienexpertise verfügten, über externe Medienexperten/innen, die für die Durchführung der Projekte engagiert wurden, bis hin zu Professionellen und Ehrenamtlichen mit geringen medienpädagogischen Kenntnissen. Manche von ihnen hatten die Geschlechterthematik im Blick, andere nicht oder nicht durchgängig. Kriterien für die Auswahl von jeweils sechs zu evaluierenden Projekten waren der regionale Kontext (Grossstadt, Mittelstadt, Kleinstadt oder ländliche Gemeinde), die Art des Projektes (Mit welchem Medium wurde gearbeitet? Welche Inhalten und Themen standen im Vordergrund? Lag der Schwerpunkt auf Mediennutzung und/oder Mediengestaltung?), die Art des Projektträgers in der Jugendhilfe-Landschaft, die angesprochenen Zielgruppen und ihre Zugangsmöglichkeiten zu Medien (Alter, soziale, familiäre und bildungsbezogene Hintergründe, vor allem auch: Migrationshintergrund ja/nein, und die Frage, ob das Projekt explizit für Mädchen oder für Jungen angeboten wurde oder auf die Teilnahme von Jungen und Mädchen zielte). Durch diese beiden Evaluationsphasen hatten wir die Möglichkeit, unterschiedliche Kombinationen von Methoden auszuprobieren. In der ersten Evaluationsphase kombinierten wir die Methoden der teilnehmenden Beobachtung ${ }^{2}$, Gruppendiskussionen mit den Jugendlichen ${ }^{3}$ und Experten/-inneninterviews mit pädagogischen Leiterinnen und Leitern zu Beginn und am Ende der Phase ${ }^{4}$. In der zweiten Evaluationsphase variierten wir den Methoden-Mix an einem Punkt: Statt der teilnehmenden Beobachtung wurden neun biographische Interviews mit Jugendlichen zu ihren Bildungserfahrungen in der Medienpädagogik geführt, die in Anlehnung an Rosenthal und anderen (2006) ausgewertet wurden. ${ }^{5}$

\section{Vorzüge und Nachteile der beiden Methodenkombinationen unter der Gender-Perspektive}

Im Rückblick auf die beiden unterschiedlichen Methodenkombinationen hatte das Einbeziehen eines ethnographischen Zugangs (teilnehmende Beobachtung) in der ersten Phase diverse Vorteile. Hierdurch erhielten wir Einblick in die realen Interaktionen zwischen den Jugendlichen, aber auch zwischen Jugendlichen und pädagogischen Leiterinnen und Leitern. Dies war gerade auch unter der GenderPerspektive spannend, weil Gender-Dynamiken in Gruppen beobachtet werden

2 Tandem-Beobachtung durch jeweils zwei Forscher/innen, Einsatz von Beobachtungsbögen, die neben der Beschreibung von Interaktionen auch die Mitschrift von Gesprächssequenzen umfassten und inhaltsanalytisch ausgewertet wurden.

3 Diese wurden entlang des Gruppendiskussionsverfahrens nach Bohnsack (2003) durchgeführt, aus forschungspragmatischen Gründen konnte die Auswertung aber nicht der dokumentarischen Methode folgen, sondern war inhaltsanalytisch ausgerichtet.

4 In Anlehnung an Meuser und Nagel (1991), unter Berücksichtigung der methodologischen Überlegungen von Pfadenhauer (2002).

5 Hierbei wurden aus den neun vollständigen Transkripten drei für eine vertiefende Auswertung ausgewählt. Letztere umfasste einen reformulierenden, einen reflektierend-hypothesengenerierenden Schritt und eine Feinanalyse von ausgewählten Textstellen, welche im Hinblick auf biographische Bildungsprozesse besonders aussagekräftig waren (vgl. Rosenthal 2005). 
konnten, die so weder in den Experten/-inneninterviews noch in den Gruppeninterviews mit Jugendlichen thematisiert worden sind. Die teilnehmende Beobachtung verschaffte ausserdem atmosphärische Eindrücke, die keine Erzählung so wiedergeben kann. Sie bewirkte ein Warm-Werden von allen an der Forschung Beteiligten und ein Vertraut-Werden der Forschenden mit den (internen) Schwierigkeiten, aber auch den Potentialen des pädagogischen Prozesses. Sie vermittelte vor allem auch eine direkte Anschauung der Arbeitsweise und der Kompetenzen der Leitenden - zum Beispiel im Umgang mit Gruppendynamiken oder im Aufgreifen von Potentialen -, die diese so niemals beschreiben würden.

Gleichzeitig bestätigten sich auch in unserer teilnehmenden Beobachtung die (wohlbekannten) Begrenzungen dieser Methode (vgl. zusammenfassend Lüders 2003): Abgesehen davon, dass die Anwesenheit der Beobachter/innen die gesamte Situation verfremdet und Wirkung hinterlässt, gibt sie immer nur punktuelle Einblicke in etwas, das eigentlich nur als Prozess wirklich verständlich ist - als Prozess mit einer unter Umständen langen Vorgeschichte (z. B. zwischen der Leiterin und der Mädchengruppe). Diese punktuellen Einblicke können den Eindruck stark verfremden: Vielleicht hat in einem vorhergehenden Projekttreffen eine spannende Interaktion stattgefunden, nicht aber in der beobachteten Situation, oder vielleicht bricht gerade die beobachtete Session aus der Interaktionsnormalität der Gruppe aus. Um der Gefahr zu begegnen, aufgrund des immer nur ausschnitthaften Teilnehmens bestimmte Situationen unter- oder überzubewerten, triangulierten wir die Auswertung der teilnehmenden Beobachtung mit Ergebnissen aus den Experten/-inneninterviews und den Gruppeninterviews mit den Jugendlichen. Doch auch mit dieser Triangulation blieb manches offen: So konnten wir nichts Genaueres darüber erfahren, welchen Stellenwert die Bildungsprozesse, die die jungen Frauen und Männer in den medienpädagogischen Projekten durchlaufen hatten, in ihrer biographischen Entwicklung bekommen haben.

Diesbezüglich haben nun rekonstruktive Verfahren, in unserem Falle (narrative) Interviews mit Nachfrageteil, die wir in der zweiten Phase der Evaluation eingesetzt hatten, ihre Vorteile. Sie liegen für die Befragten darin, dass sie den Reflexionsraum nutzen können, der durch das Interview entsteht, und von dem sie unserer Erfahrung nach sehr gerne Gebrauch machten. Diese Gelegenheit wird auch von Jugendlichen mit geringeren Sprachkompetenzen genutzt - hier bestätigten sich die methodologischen Überlegungen von Gabriele Rosenthal und anderen (Rosenthal u. a. 2006) zu biographischen Interviews mit problembelasteten Jugendlichen. Indem Jugendliche ihre medienpädagogischen Erfahrungen in ihre biographische Rekonstruktion integrierten, wurden die Interviews selbst - als Reflexions- und ReKonstruktionsräume - zu potentiellen Bildungsräumen.

Deutlich wurde dabei auf der inhaltlichen Ebene die Situiertheit, das heisst der konkrete lebensgeschichtliche Kontext der Bildungserfahrungen: Die medienpädagogische Erfahrung findet nie isoliert statt, und für die Untersuchung von sol- 
chen Bildungsprozessen ist es daher immer wichtig, das, was im Umfeld solcher Projekte passiert, mit einzubeziehen. Vor allem in der Jugendhilfe, aber nicht nur hier, ist es von Interesse, inwieweit Erfahrungen und (Reflexions-)Prozesse im lebensweltlichen Kontext durch konkrete medienpädagogische Projekterfahrungen angestossen werden - und umgekehrt: inwieweit Erstere die Medienarbeit anregen und inspirieren und zu einem Feld der Aufarbeitung und Bewältigung von Erfahrungen im lebensweltlichen Kontext werden lassen. ${ }^{6}$

Nun schaffen Gruppen- oder Einzelinterviews, hier hat unsere Erfahrung die Praxis der medienpädagogischen Forschung bestätigt (vgl. Niesyto 2007), gerade zu solchen Ereignissen im Umfeld der Medienproduktion, die durch eine teilnehmende Beobachtung nur schwer erfasst werden könnten (es sei denn, es handelte sich um eine konsequente Prozessbegleitung) geeignete methodische Zugänge. Besonders hervorheben wollen wir hier die Qualitäten eines narrativen Zugangs.

Ein Beispiel hierfür ist das biographische Interview mit Reyhan ${ }^{7}$. Die 14-Jährige hat im Rahmen des Ganztagsangebots einer grossstädtischen Hauptschule an einem Videoprojekt teilgenommen - durchgeführt von einem Mädchentreff im Stadtteil, den sie ohnehin häufig besucht. Reyhan rekonstruiert in ihrer Erzählung einen zentralen Reflexionsprozess, der angestossen wurde durch das Motto des Filmwettbewerbs, an dem das Videoprojekt teilnahm. Die Geschichte, die die Mädchengruppe (fünf 12- bis 14-jährige Hauptschülerinnen türkischer Herkunft) sich hierzu ausdachte, thematisierte die Spannung zwischen Dazugehören-Wollen und Ausgeschlossen-Werden am Beispiel einer neuen Schülerin in einer Schulklasse: ‘Die Neues ist anders, sie trägt geschlossene Kleidung und Kopftuch und ist sehr gut in der Schule. Sie wird von den anderen gehänselt und gleichzeitig beneidet. Da sie unbedingt dazugehören will, klaut sie in einem Laden ‘offene` Klamotten und zieht sich jedes Mal heimlich vor der Schule um - bis sie von ihrem Vater entdeckt wird. Reyhan erzählt, wie die Mädchengruppe, um diese Klau-Szene drehen zu können, einen Ladenbesitzer um Dreherlaubnis fragen musste - um sich dann seinen Vorwurf gefallen zu lassen, mit ihrem Film überkommene Klischees zu reproduzieren:

Der ... also der war selbst Türke; und wir haben ja so eine türkische Familie gemacht. Er fand es, glaube ich, als erstes so, wie wir es erklärt haben, irgendwie komisch. Also er hat gemeint «ja so sind wir eigentlich gar nicht immerı, ja? Weil ich, glaub', das hat ihm irgendwie auch schon ein bisschen genervt, weil wir so ... so türkische Familie, so streng ... ich bin auch selbst ... ich bin auch selbst Türkin und mein Vater ist nicht so, aber wir wollen halt so eine Situation wie die anderen Leute eigentlich so

\footnotetext{
6 Es ist nachgerade ein Qualitätskriterium der meisten ausgewählten Projekte, eine Transferproblematik zwischen Lernerfahrungen im Medienprojekt und den sonstigen Lern-Umwelten gar nicht erst aufkommen zu lassen, sondern das jeweilige medienpädagogische Angebot in bestehende Erfahrungsräume zu integrieren.

7 Pseudonym wie alle weiteren Namen auch.
} 
denken, ja? Und er hat gemeint, das sollten wir eigentlich nicht machen, aber dann haben wir halt so erzählt, dann haben wir auch das Motto gesagt und haben erzählt, dass halt die Geschichte uns so eingefallen ist, weil so viele so drüber denken, ja? (...) Der war selbst Türke und hat ... der hat gemeint, <ich bin halt nicht so, nicht jeder Türke ist so s. Es sind auch verschiedene, auch deutsche oder so, die bis heute so denken und so streng sind. (Reyhan/B/10) ${ }^{8}$

Der Reflexionsprozess - hier: sich mit der Produktion von Klischees und mit der falsch verallgemeinernden Wirkung medialer Bilder auseinander zu setzen - findet in diesem Fall im Umfeld der Videoproduktion statt. Weil im Interview der ganze Kontext und die ganze Vorgeschichte des Projekts zur Sprache kommen kann, kann er hier auch rekonstruiert werden.

Nun wird ja - z. B. von Horst Niesyto (2001) - zurecht kritisch auf die Begrenzungen von Verfahren hingewiesen, die einseitig auf Verbalisierung(sfähigkeit) abstellen, und auf überzeugende Weise vorgeführt, wie diese Einseitigkeit durch anderen, stärker den medialen (Selbst-)Ausdruck einbeziehenden Verfahren vermieden werden kann (vgl. hierzu auch Holzwarth 2006 sowie zahlreiche Beiträge dieser Tagung). Jede Methode hat ihre Begrenzungen, und jede Methode prästrukturiert die Identitätskonstruktionen, die mit ihr generiert werden (vgl. Holzwarth in diesem Band) - eine Tatsache, der sich Forscher und Forscherinnen bewusst sein und die sie auch benennen müssen (vgl. Ahmed 2007). Doch jede Methode hat auch gewisse Vorteile. Im Hinblick darauf, dass in der Medienpädagogik oft Reflexionsräume zur Aneignung des Erlebten fehlen, haben Gespräche sogar ganz genau bestimmbare Vorteile, die in der medienpädagogischen Forschung ja auch genutzt werden (vgl. Niesyto 2007): So konnten wir in diesen Interviews feststellen, wie Erlebnisse aus den Projekten rückblickend verarbeitet und womöglich erst im Gespräch hierüber zur reflektierten Erfahrungen werden. Häufig in den Interviews zu hörende Äusserungen wie: «hab ich mir noch gar nie so klargemacht» geben hiervon Zeugnis. Unter der Gender-Perspektive sind hierbei die Anstösse, die die Erfahrungen in den Medienprojekten für die individuelle Persönlichkeitsentwicklung (als junge Frau, als junger Mann) geben können, besonders interessant. So wurde zum Teil von einem veränderten Welt- und Selbstverhältnis durch die medienpädagogische Erfahrung gesprochen, von veränderten beruflichen Perspektiven und damit verbundenen Lebensentwürfen (vgl. das biographische Portrait von Selam in Huber u.a. 2007). Reflektierende Gespräche ermöglichen die Rekonstruktion von biographischen Bedeutungen, die die Medienarbeit haben kann, und über diese Rekonstruktion kann Bildungsprozessen im Geschlechterbezug auf die Spur gekommen werden.

8 Abkürzung ist so zu lesen: Zitat Reyhan, Biographisches Interview, S. 10 des Transkripts. 
Freilich ist hier wiederum zu beachten, dass nicht nur das interessant ist, was Jugendliche reflektieren, sondern auch das, was sie nicht reflektieren, und wodurch sie indirekt Einblick geben in die Welt ihrer Selbstverständlichkeiten und Normalitäten'. So werden viele Aspekte der Mediennutzung nicht (mehr) thematisiert, da sie zu selbstverständlichen Bestandteilen des jugendlichen Alltags geworden sind. Dieses Beispiel verweist einmal mehr auf die Notwendigkeit, im thematischen Kontext von Medien mit einer besonderen Sensibilität für generationengebundene Nutzungsgewohnheiten und -normalitäten vorzugehen.

Sogar auf der Ebene einer Rekonstruktion des Erwerbs von Medienkompetenz (vgl. Baacke 1999) boten Interviews zumindest hilfreiche Ergänzungen zur direkten Beobachtung praktizierter Medienkompetenz im pädagogischen Prozess. So berichteten junge Frauen und Männer, wie ihre Wahrnehmungsfähigkeit, ihre mediale Decodierungsfähigkeit, ihre Analyse-, Reflexions- und Urteilsfähigkeit in den Projekten geschärft wurden. Zum Beispiel beschrieben sie anschaulich, wie sich durch unterschiedliche Kamerapositionen auf dieselbe Situation völlig unterschiedliche Aussagen transportieren lassen, rekonstruierten also diesen Lernprozess im Interview. Auch hier gab es immer wieder interessante Bezüge zur Gender-Thematik: So konnte in Interviews erschlossen werden, wie Medienkompetenz und Geschlechterkompetenz - im Sinne einer kritischen Auseinandersetzung mit Bildlichkeit und ihren Zuschreibungen - zusammenhängen (vgl. Stauber/Kaschuba 2006).

Hierbei klingt an, was im Kontext einer emanzipatorischen Medienpädagogik für wichtig gehalten wird, nämlich nicht nur die vorhandenen Themen, Ausdrucksformen und Mediennutzungsstilen von Kindern und Jugendlichen anzuerkennen, sondern auch eine «selbstreflexive und kritische Auseinandersetzung mit den Medien zu intensivieren und Medienkritik noch stärker mit den Aufgaben der Persönlichkeitsbildung, der sozialen Milieubildung und einer qualitätsbezogenen Auseinandersetzung mit Medienangeboten zu verbinden» (Niesyto 2004: 8).

Hier bestätigt sich, dass eine solche emanzipatorische Medienpädagogik genau auch das Gespräch, und die Evaluation derselben genau auch das Interview brauchen - also sprachlich vermittelte Formen des Reflektierens, die den Selbstausdruck mit Medien nicht ersetzen, aber in seiner individuell biographischen und seiner kollektiv-gruppenbezogenen Bedeutung ausleuchten (vgl. Niesyto 2007). In einem Interview, das Peter Holzwarth mit David Gauntlett geführt hat, wird die Nähe zwischen den «visual methods» und dem Reflektieren eines durchlebten (medienpädagogischen) Prozesses sehr deutlich. Auf die Frage Holzwarths what about the view that generating some speech, in an interview or focus group, is also a creative act - just as creative as the methods you are talking about?

antwortet Gauntlett:

\footnotetext{
9 Vgl. zum dokumentarischen Sinngehalt Bohnsack 2003.
} 
Well it's true that talk is creative too, yes, of course. But the reflective process of making an artefact, taking time, as well as the act of making something that you can look at and think about and change, is different. First, you're asked to make the thing - which might be a drawing, or collage, or video, or Lego model, or whatever - and you could think about it quite normally for ten or fifteen minutes, say, or even a couple of days or weeks, depending on the study, and then you would make the thing, and again that takes time and involves constant engagement with the artefact. By the time you're at the end of that process ... it's quite different to being in a face-to-face session where somebody asks a question and you have to provide an answer straight away. (...) If the body is central to, or even just an important dimension of, experience - which I think it is - and if people's own creative, reflexive responses to things are important - which I think they are - then we need to work with people in ways that embrace this, rather than ignore it. (Gauntlett 2006:7)

Nun ist die in unseren Evaluationen favorisierte und praktizierte Art des narrativen Interviews von dieser die körperliche Erfahrungsdimension einbeziehenden Methode gar nicht weit entfernt: Dieses Interview findet statt nach Abschluss eines längeren medienpädagogischen Projektes, das eine Fülle von Erfahrungen lieferte, und die zur Reflexion aufgeforderte Person hat nun die Möglichkeit, entweder den Produktionsprozess und seine Bedingungen, oder das Produkt, oder Prozesse im Umfeld der Produktion zum Aufhänger für die Rekonstruktion aller Arten von subjektiven Relevanzen zu machen. Ein kreativer Akt, der das Produkt entweder direkt vor Augen führt oder im Gedächtnis wieder aufleben lässt, und anhand dessen sie oder er die hieran festzumachenden Bildungsprozesse rekonstruieren kann. Dieser Rekonstruktionsprozess kann noch stärker im Sinne einer productelicitation angelegt werden.

Unterschiedliche Interviewverfahren schaffen unterschiedliche RekonstruktionsRäume. So hat das Einzelinterview oft gegenüber der Gruppendiskussion den Vorteil, den Stimmen der befragten Jugendlichen mehr Gehör zu verleihen, als dies in der Dynamik der Gruppendiskussionen möglich ist, ihnen vor allem auch Gelegenheit für tiefergehende Reflexionen zu geben. Andererseits kommen in der (gewünschten) Selbstläufigkeit der Gruppendiskussion Themen zur Sprache, die junge Frauen und Männer im Einzelgespräch vielleicht ausklammern würden - was die Chance einer Themenöffnung birgt, genauso aber die Gefahr, dass individuelle Grenzen überschritten werden. Oder umgekehrt: Prozesse werden im Einzelgespräch auf ihre Hintergründe und Vorgeschichten beleuchtet, die im Gruppengespräch leicht übergangen werden können. Bei Methodenkombinationen, wie wir sie angewendet haben, heben sich die Effekte der verschiedenen Interviewver- 
fahren zwar nicht auf, relativieren sich jedoch durch die Möglichkeit, triangulierend in dem jeweils anderen Verfahren zu anderen Erkenntnissen zu kommen.

\section{Einige Ergebnisse zur Relevanz einer Berücksichtigung von Gender-Dynamiken in medienpädagogischen Settings}

Wichtig war uns in beiden Evaluationsphasen ein rekonstruktiver Zugang auf das Thema: Es ging uns darum, unterschiedlichen Ebenen gesellschaftlicher Zuschreibungen - etwa aufgrund von Geschlecht und Ethnizität - auf den Grund zu gehen und aufzuzeigen, wo und wie es durch Medienprojekte gelingt, diese kritisch zu hinterfragen. Die dabei eingesetzten rekonstruktiven Forschungsmethoden sind zwar auch nicht davor gefeit, Zuschreibungen vorzunehmen. Aber schon allein ein Perspektivenwechsel von der Suche nach Unterschieden zwischen Mädchen und Jungen im Umgang mit Neuen Medien hin zu den Bedeutungen und Handlungsmöglichkeiten, die Medien für Mädchen und für Jungen jeweils beinhalten, schafft Raum für die eigenen und eigenwilligen Bilder und Vorstellungen der Mädchen und Jungen selbst, aber auch der erwachsenen Medienpädagogen und -pädagoginnen.

Im Folgenden greife ich einige zentrale Ergebnisse im Hinblick auf Gender-Dynamiken heraus, die wir in den Medienprojekten, in den konkreten Erhebungssituationen und in den Rekonstruktionen der Jugendlichen identifizieren konnten. Ich unterscheide zwischen Gender-Dynamiken in den Medienprojekten, die sich direkt auf den Erhebungsprozess auswirken, Gender-Dynamiken in der Rekonstruktion der Jugendlichen, und Dynamiken, die die Betonung der Gender-Thematik für die Forschungs-Interaktion (z. B. wie hier in Experten/innen-Interviews) freisetzen kann. Alle Beispiele entstammen der zweiten Evaluationsphase (siehe Huber u. a. 2007).

\section{Gender- Dynamiken in den Medienprojekten: Das Beispiel CHAT}

CHAT, ein Projekt zur Vorbereitung eines Beratungschats für Jugendliche zu Fragen von Sexualität und Verhütung, durchgeführt von den Mitarbeiterinnen einer Familienberatungsstelle mit der 9. Klasse eines Ganztagsgymnasiums im ländlichen Raum, hatte zwar in seinem Entwurf Geschlechterbezüge mitgedacht, in der Umsetzung jedoch die Gender-Dynamiken innerhalb der Klasse in Zusammenhang mit dem Medium der Online-Beratung nicht immer hinreichend in Betracht gezogen. Als die Klasse innerhalb des Projekts gefragt wurde, wer sich nach den Vorbereitungen im Rahmen des Projekts an der Schule und der Auseinandersetzung mit Chats mit der längerfristigen Umsetzung des Beratungschats beschäftigen möchte, zeigte sich eine Gruppe interessiert, die ausschliesslich aus Mädchen bestand. Auf die Frage, wie sich die Mädchen die Zusammensetzung der Gruppe erklären können, beschreiben diese den Vorgang der Gruppenfindung aus ihrer Sicht: 
M1: Also die zwei Frauen haben uns aufgeteilt, und haben dann auch gefragt, wie wir's fanden und haben dann gesagt, ja wer Interesse hätte, soll seinen Namen aufschreiben. Es haben, glaub', nur Mädchen ihren Namen aufgeschrieben. Von den Jungen hatte, glaub', gar keiner Lust, sich da weiter reinzuhängen oder so.

M2: Es war auch so, wir sassen da, glaub', auf einer Wiese, und dann hat sie uns gefragt. Da sassen wir draussen auf dem Schulhof, da hat sie uns gefragt, wer da so weiter machen will. Hat sie uns dann noch mal gefragt. Und ich glaube, dass sich keiner von den Jungen ... sich getraut hat, da irgendwie weiter zu machen. Weil sich das natürlich rumspricht. Und ich glaub', für die ist das eher peinlich, so was zu machen mit so Problemen und so. Das finden die eher lächerlich wie jetzt sinnvoll. Also so eine Aussage ...

M1: ... oder sagen's nicht, wenn sie's sinnvoll finden.

M2: Ja ... ich denke, manche hätten's vielleicht schon gemacht, wenn's irgendwie ...

I: Wenn's irgendwie?

M2: Ja, also das kann man ja nicht geheim halten sozusagen. Und ich glaub, dass sie dann irgendwie gedacht haben, «buoah, ist ja lächerlich» oder so.

M1: Und dass sie dann von den anderen Jungen dann irgendwelche blöden Kommentare abkriegen oder so. (CHAT/GD/12/M1+2+I) ${ }^{10}$

Für die beiden Mädchen liegt es also auf der Hand: Wenn, wie es hier der Fall war, für ein solches Projekt in der Öffentlichkeit der Klassengemeinschaft geworben wird, dann werden sich die Jungen nicht melden, auch wenn durchaus die Möglichkeit einer Öffnung dieser Rollen besteht: «... manche (Jungen) hätten's vielleicht schon gemacht ...». Ihrer Einschätzung nach fällt es den Jungen im Kontext einer Schul-Öffentlichkeit, die in traditioneller Weise wertende Geschlechterzuschreibungen vornimmt, schwer (und ihnen als Mädchen entsprechend leicht), sich öffentlich zur Beschäftigung mit persönlichen Themen zu bekennen. Die Leiterinnen des Projekts hatten diese Gender-Dynamiken nicht im Blick, wodurch sich die zugeschriebenen Geschlechter-Rollen in der Gruppe manifestierten.

Obwohl dieses Interview im Hinblick auf die unterschiedlichen Umgangsweisen der Schülerinnen und Schüler mit geschlechterbezogenen Zuschreibungen äusserst aufschlussreich war, so hatte freilich die stattgefundene Aufteilung der Klasse zur Folge, dass für die Evaluation des weiteren Projektverlaufs nur die Stimmen der Mädchen, nicht jedoch die Haltungen und Meinungen der Jungen weiter beleuchtet werden konnten.

10 Abkürzung ist so zu lesen: Zitat aus dem Kontext des Projekts CHAT, Gruppendiskussion, S. 12 des Transkripts, Mädchen 1 und 2 und Interviewerin. 


\section{Gender-Dynamiken in den Rekonstruktionen der Jugendlichen}

Auch in den Rekonstruktionen der Jungen und Mädchen finden sich Gender-Dynamiken, z. B. biografische (Um-)Orientierungen und Entwicklungen, in die Geschlechterkonstrukte hineinspielen - auch in dem Sinne, dass sie als Kontrastfolie für anstehende Entscheidungen dienen. So orientiert sich Selam, eine Gymnasiastin, aufgrund der Erfahrungen in einem Videoprojekt neu: Zum Zeitpunkt des Interviews, das nach Abschluss des Medienprojektes geführt wird, ist sie dabei, sich von Betriebswirtschaftslehre auf ein Medienfach umzuorientieren, was ihr (und ihrer eritreischen Familie) andere Geschlechter-Konnotationen ermöglicht bzw. abverlangt. Verallgemeinernd lässt sich sagen: Dadurch, dass Bildungsprozesse immer situiert sind, und zwar zumeist in einem subtil oder offensichtlich ge-genderten Setting, sind sie einbezogen in diese Gender-Dynamiken.

Ein weiteres Beispiel ist Carsten: Der Kontext ist hier ein Medienprojekt einer Einrichtung der erzieherischen Hilfen im Stadtteil, in dem es um das Erlernen von Grundkenntnissen in den Programmen Word und Excel geht. Dies wird umgesetzt anhand eines Fragebogens zum Thema "Gewalt in den Lebenswelten der Jugendlichen», den die Jugendlichen - zusammen mit ihrem Leiter - entwickelt hatten, anhand dessen sie Klassenzimmerbefragungen und -diskussionen durchführten und den sie anschliessend auswerteten. Für Carsten, der als Streitschlichter früher an der Schule schon viel soziale Verantwortung übernommen hat, ist dieses Projekt eine Gelegenheit zur Verarbeitung eigener (Gewalt-)Erfahrungen:

I: Wie hast du denn das Projekt so für dich erlebt?

$\mathrm{J}$ : Persönlich. Ein paar Sachen waren angreifend. Also wenn ich jetzt irgendwie 'nen Bogen durchgelesen hab, mir gedacht hab, «hei, das passt zu dir', dann hab' ich mir schon ab und zu im Hinterkopf gedacht «was für Probleme hat der noch?> (...) Und deswegen. Haben wir (...) auch aus persönlichen Gründen ... haben wir auch bei manchen Bögen gesagt, «du ich kann hier nicht mehr weitermachen, machst du den weiter> oder so. Also wenn da jetzt irgendwie was drinne stand ... (...) Das ging eigentlich so an die Grenzen. (...) Ich glaube, das ging mehreren so. Ab und zu sind die Bögen nur so im Kreis gewandert. (...) Also wirklich, der eine hat angefangen und bei Seite 8 nicht mehr, hat gesagt: «Kannst du weitermachen bei Seite 8? und dann ging's bei mir an Seite 10 zum Beispiel, weil da wieder was war, was mich an früher erinnert hat und dann hab' ich's wieder an jemand weitergegeben. (Carsten/B/47-48)

Im Projektverlauf bestätigte sich also, dass er mit diesen Gewalterfahrungen nicht alleine ist, sondern die Mädchen und Jungen seiner Gruppe bei der Auswertung der Fragebögen immer wieder konfrontiert waren mit ihrer eigenen Geschichte. 
Nun hatten die bisherigen Aktivitäten dieses Stadtteil-Projekts immer auch das Ziel, in der Öffentlichkeit zu wirken. Diesmal jedoch will Carsten sich nicht auf die bisher übliche Weise an der Öffentlichkeitsarbeit beteiligen:

«es gibt viele Leute, die meinen dann «ja warum hat er das gemacht? Der liebt Gewalt. Nur deswegen hat er ein Projekt gemacht.ı» (Carsten/B/63)

Seine Begründung: Die anderen Jugendlichen würden ihm unterstellen, er suche nur nach irgendeiner Form, sich mit dem Thema Gewalt zu befassen. Als Erfahrungshintergrund benennt er seine Zeit als Streitschlichter an der Schule, wo er mit solchen Zuschreibungen bereits konfrontiert war. Carsten setzt sich somit mit einer (nur im Gender-Bezug zu verstehenden) (vermeintlichen) Unterstellung seiner peers auseinander. Er erkennt hier die Gefahr von falschen Zuschreibungen und versucht präventiv, sein Verhalten hierauf einzustellen. Dabei inszeniert er sich wie so oft in diesem Interview in deutlicher Abgrenzung zu diesem JugendlichenVerhalten.

\section{Die Gender-Thematik als Freisetzung von Dynamiken in der Forschungs-Interaktion}

Das Benennen eines geschlechterbezogenen Forschungsinteresses, zum Beispiel eines Interesses daran, wie der Erwerb von Medienkompetenz mit dem Erwerb von Gender-Kompetenz zusammenhängt, kann in manchen Forschungssituationen und -interaktionen als Türöffner wirken. Dies konnten wir zum Beispiel in einem Experten/inneninterview mit Leiterinnen eines Videoprojekts für Mädchen feststellen. Hier entstand ein Gespräch über Gender-Kompetenz im Zusammenhang mit der Erweiterung von Horizonten - durch Vorbilder und alternative Lebensentwürfe. Angeregt durch diese Thematisierung der Gender-Perspektive unserer Forschung erzählt die junge Medienpädagogin des Video-Projekts CLIP von Situationen, die eher am Rande des Medienprojektes entstanden, in dem sie mit 12- bis 14-jährigen Mädchen (überwiegend mit türkischem Hintergrund) gearbeitet hatte:

Also (...) was für die ja auch immer ganz spannend ist, die Frau, die sie vor sich haben, also die war halt ich, was macht denn die so. Also, (...) ich bin da immer so ein bisschen rausgefallen, also ich bin 'ne Frau Anfang dreissig, die nicht verheiratet ist und keine Kinder hat, ich bin teilweise [älter als, A.d.V.] deren Mütter ... Ah, ich lebe allein, ich hab' 'nen Beruf und bin selbständig. Das war für die schon mal so was, was eigentlich in ihrer Realität nicht so vorkommt. Und ich hab auch noch kurze Haare und bin trotzdem total nett. Also das war für die irgendwie (...) ganz arg spannend, und haben sie immer ganz viel gefragt. Und dann hab' ich schon auch gemerkt 〈au〉, dann haben sie auch öfter mal einfach so einen kurzen Ausflug gemacht und gesagt «Mensch, wie stellt ihr euch das mal 
vor?> und das war für die ganz spannend, auch mal was anderes einfach mitzukriegen. ¿Was, du wohnst allein?» und ‘oh, ah, ja das find ich auch mal, toll!) Also die sind jetzt schon auch mal ins Grübeln gekommen. Ob das nicht auch was für sie sein könnte, oder ... Ich mein', die sind jetzt 6. Klasse und sind noch weit davon entfernt, aber (...) die sind ja oftmals realitätsnaher als, sag ich mal, als Mädchen in dem Alter, die jetzt in ganz behüteten Verhältnissen aufwachsen, von daher glaubs ich, ist das schon auch ein bisschen früher Thema bei denen. (CLIP/E2/9/F)

Wichtig ist hier, dass die Leiterin diese Prozesse als relevante Bildungsprozesse anerkennt und auch rekonstruiert. Und dass sie Einblick gibt in ihre (geschlechterpädagogische) Praxis, in der sie die produktive Irritation der Mädchen wahrnimmt und als Anlass nutzt, um an den passenden Stellen hierzu den Raum für Gespräch und Reflexion zu öffnen. Das heisst: Sie hat - im Interview und in ihrer Praxis - nicht nur das Gelingen des Videoprojekts im Blick, sondern auch die Interessen der Mädchen, sich mit Fragen von weiblichen Lebensentwürfen auseinanderzusetzen. Dass sie hiervon überhaupt berichtet, führen wir darauf zurück, dass sie bei den Fragenden begründeterweise ein Interesse an solchen vermeintlichen «Nebenschauplätzen» für Geschlechter-Lernen vermuten kann. So erzählt sich auch, wie die Mädchen mit dem Medium Video aktiv die Möglichkeit genutzt haben, sich zum Beispiel beim alltäglichen Blödeln aufzunehmen:

Ich hatte auch den Eindruck, das Angucken war auch ganz arg wichtig. (...) Wie wirke ich, wenn ich so und so bin, was ich nie sein darf? (...) Also so hab ich manchmal das Gefühl, natürlich, klar, diese Szene wird verworfen, aber ich habs immer so den Eindruck gehabt, die wird noch mit viel mehr Spannung angeguckt bei den Mädels, wie die Szenen, die dann hinterher im Film kommen. (...) Also dass die eigentlich die wichtigeren Szenen auch waren. (...) Wo sie sich ausgetobt und ein Stück weit vielleicht auch irgendwelche Illusionen oder Wünsche oder Träume irgendwie dann rüberkommen oder so Fantasien. (CLIP/E2/20/F)

Auch diese Aussage wird durch einen Interaktions-Kontext, in dem eine Verständigung über die Relevanz sämtlicher Lernerfahrungen stattgefunden hat, befördert. So rückt die Art und Weise, wie die Mädchen das Medium Video nutzen, um unterschiedliche Selbstinszenierungen auszuprobieren, als wichtiger Experimentierraum ins Blickfeld. Dies ist hier mit «Freisetzung von gender-relevanten Themen` gemeint.

Nun wird ein solches Herangehen schnell mit dem Vorwurf konfrontiert, Geschlecht zu reifizieren - also genau das aufzufinden, wonach unter einer bestimmten Forschungsperspektive gesucht wird. Dieser Vorwurf ist im Hinblick auf Geschlechter- 
differenz oft berechtigt: Tatsächlich kann unter dem Fokus «Differenz» sehr schnell das reproduziert werden, was möglichst unbefangen` untersucht werden sollte (vgl. zum Vorwurf der methodisch bedingten Reifikation des Systems der Zweigeschlechtlichkeit Gildemeister/Wetterer 1992; Hagemann-White 1993; Gahleitner 2004). Nun ist aber die Frage: "Was ist hieran unter der Gender-Perspektive interessant?» (also die schiere, inhaltlich offene Thematisierung eines Interesses) nicht zu verwechseln mit der Frage: «Was ist hieran im Hinblick auf Geschlechterunterschiede interessant?» So muss der Reifikations-Vorwurf zum einen genau seinen Gegenstand überprüfen, und immer wieder auch auf sich selbst angewendet werden: Inwieweit unterstellt er (quasi automatisch), mit der Thematisierung von gender-bezogenen Forschungsinteressen sei die Suche nach Bestätigung des Differenzgedankens verbunden? Wenn ich als Forscherin - zumal in einem Experten/-innen-Interview «auf gleicher Augenhöhe» - mein Interesse an bestimmten Fragestellungen offenlege, dann bedeutet das nicht automatisch, bei meinen Interviewpartnern/-innen eine "Überfokussierung» zu bewirken. Allerdings, und hierfür sensibilisiert der Reifizierungsvorwurf, ist genau zu überprüfen, in welche Richtung denn die Thematisierung von Geschlecht verstanden wurde - und gegebenenfalls bestimmte (die Differenz reifizierende) Missverständnisse auszuräumen. Im Interview mit der Leiterin von CLIP gab es diesbezüglich jedenfalls kein Missverständnis: Sie blieb konsequent auf der Ebene der Rekonstruktion der Praktiken ihrer Projekt-Teilnehmerinnen und auf den Interaktionen zwischen ihr und den Mädchen. Sie beschrieb und erklärte sich (und uns) informelle Lernprozesse im Kontext des non-formalen Bildungsraumes «Videoprojekt» - darin bestärkt, dass genau solche Prozesse gerade unter einer Gender-Perspektive interessant sind. Denn diese fragt danach, wie sich Mädchen in ihren Entwicklungen Vergewisserung und Orientierung organisieren, wie sie pädagogische Angebote hierfür (ungeplanterweise) nutzen, wie sie sich hierfür Raum verschaffen. Damit sind keinerlei Aussagen über «Geschlechts-Spezifika» oder «Differenzen» getroffen.

Aus diesen exemplarischen Ergebnissen lassen sich folgende Schlussfolgerungen für eine Berücksichtigung von Gender-Dynamiken in der medienpädagogischen Forschung und Praxis ziehen:

Zum einen sind die jeweiligen medienpädagogischen Settings daraufhin zu überprüfen, inwieweit sie solche Gender-Dynamiken berücksichtigen. Denn es ist davon auszugehen, dass dadurch sowohl in der Praxis als auch für die Forschung bessere Zugangsmöglichkeiten zu Mädchen und Jungen entstehen. Zum zweiten ist auf der Ebene der Medienbildung der Erwerb von Medienkompetenz um die Dimension der Gender-Kompetenz zu erweitern - auch dies eine Folgerung, die nicht nur die Forschung, sondern auch die pädagogische Praxis sensibilisieren kann. Schliesslich erweitert die Berücksichtigung von Gender-Dynamiken auf der Forschungsebene das Wahrnehmungsrepertoire dafür, was in Medienprojekten passiert, und wie sich dies in den Rekonstruktionen von Befragten widerspiegelt. 
Hierbei ist von einem Wechselspiel zwischen der Thematisierung der Forschungsperspektive und der Benennung relevanter Gender-Prozesse durch die Pädagogen und Pädagoginnen auszugehen: Oft wird letzteres erst über ersteres freigesetzt. Dies jedoch erfordert, sensibel mit dem Problem der Reifizierung von Geschlecht umzugehen und stattdessen konsequent den Blick auf Herstellungsweisen aller möglichen Geschlechterbezüge (um)zulenken.

\section{Literatur}

Ahmed, Sarina. Methodological Implications of Video as an Instrument in Qualitative Social Research. Master Thesis report for the University of London Institute of Education, School of Culture, Language \& Communication, 2007.

Baacke, Dieter. «Im Datennetz: Medienkompetenz (nicht nur) für Kinder und Jugendliche als pädagogische Herausforderung.» Ins Netz gegangen: Internet und Multimedia in der ausserschulischen Pädagogik. Hrsg. v. Vorstand der Gesellschaft für Medienpädagogik und Kommunikationskultur in der Bundesrepublik Deutschland (GMK) e. V. Bielefeld: GMK, 1999. 14-28.

Bohnsack, Ralf. Rekonstruktive Sozialforschung: Einführung in qualitative Methoden. 5. Aufl. Opladen: UTB, 2003.

Gahleitner, Silke Birgitta. «Zwischen Differenz und Dekonstruktion: Methodische Überlegungen zur Überschreitung des biploraren Geschlechterdualismus in der Genderforschung nach einem Verfahren von HagemannWhite.» Gender methodologisch: Empirische Forschung in der Informationsgesellschaft vor neuen Herausforderungen. Hrsg. v. Sylvia Buchen, Cornelia Helfferich u. Maja S. Maier. Wiesbaden: VS Verlag für Sozialwissenschaften, 2004. 283-291.

Gauntlett, David; Holzwarth, Peter. "Creative and visual methods for exploring identities.» Visual Studies, Vol. 21, No. 1 (April 2006): 82-93.

Gildemeister, Regine; Wetterer, Angelika. «Wie Geschlechter gemacht werden: Die Soziale Konstruktion der Zweigeschlechtlichkeit und ihre Reifizierung in der Frauenforschung.» Traditionen Brüche: Entwicklungen feministischer Theorie. Hrsg. v. Gudrun-Axeli Knapp u. Angelika Wetterer. Freiburg/Brsg.: Kore, 1992. 201-254.

Hagemann-White, Carol. «Die Konstrukteure des Geschlechts auf frischer Tat ertappen? Methodische Konsequenzen einer theoretischen Einsicht.» Feministische Studien, Jg. 12, Heft 2 (1993): 68-78.

Holzwarth, Peter. «Fotografie als visueller Zugang zu Lebenswelten von Kindern und Jugendlichen mit Migrationshintergrund.» Bildinterpretation und Bildverstehen: Methodische Ansätze aus sozialwissenschaftlicher, kunst- und medienpädagogischer Perspektive. Hrsg. v. Winfried Marotzki u. Horst Niesyto. Wiesbaden: VS Verlag für Sozialwissenschaften, 2006. 175-205. 
Huber, Helga; Kaschuba, Gerrit; Stauber, Barbara. Jugend und verantwortungsvolle Mediennutzung - Medien und Persönlichkeitsentwicklung. Untersuchungsbericht des Programms der Landesstiftung Baden-Württemberg. Stuttgart: Landesstiftung Baden-Württemberg, 2005.

Huber, Helga; Kaschuba, Gerrit; Stauber, Barbara. Jugend und verantwortungsvolle Mediennutzung - Medien und Gesellschaft. Untersuchungsbericht des Programms der Landesstiftung Baden-Württemberg. Stuttgart: Landesstiftung Baden-Württemberg, 2007.

Lüders, Christian. "Teilnehmende Beobachtung.» Hauptbegriffe Qualitativer Sozialforschung. Hrsg. v. Ralf Bohnsack, Winfried Marotzki u. Michael Meuser. Opladen: Leske+Budrich, 2003. 151-153.

Meuser, Michael; Nagel, Ulrike. «Experten/-inneninterviews - vielfach erprobt, wenig bedacht. Ein Beitrag zur qualitativen Methodendiskussion.» Qualitativ-empirische Sozialforschung: Konzepte, Methoden, Analysen. Hrsg. v. Detlef Garz u. Klaus Kraimer. Opladen: Westdeutscher Verlag, 1991. 441-471.

Niesyto, Horst. «Jugendforschung mit Video: Formen, Projekte und Perspektiven eines Forschungsansatzes.» Selbstausdruck mit Medien: Eigenproduktionen mit Medien als Gegenstand der Kindheits- und Jugendforschung. Hrsg. v. Horst Niesyto. München: KoPäd, 2001. 89-102.

Niesyto, Horst. «Mediale Aufmerksamkeitserregung und Medienkritik.» Ludwigsburger Beiträge zur Medienpädagogik 6 (2004): 〈http://www.ph-ludwigsburg.de/fileadmin/subsites/1b-mpxx-t-01/user_files/Online-Magazin/Ausgabe6/Niesyto6.pdf> (15.11.07).

Niesyto, Horst. «Medienpädagogische Forschung auf der Grundlage handlungsorientierter Medienarbeit.» merz: zeitschrift für medienpädagogik 5 (2006): 29-37.

Niesyto, Horst. «Eigenproduktionen mit Medien als Gegenstand medienpädagogischer Praxisforschung.» Jahrbuch Medienpädagogik 6 - Standortbestimmung der Medienpädagogik. Hrsg. v. Werner Sesink, Michael Kerres u. Heinz Moser. Wiesbaden: VS-Verlag, 2007.

Pfadenhauer, Michaela. "Auf gleicher Augenhöhe reden: Das Experteninterview - ein Gespräch zwischen Experte und Quasi-Experte.» Das Experteninterview: Theorie, Methode, Anwendung. Hrsg. v. Alexander Bogner, Beate Littig u. Wolfgang Menz. Opladen: Leske + Budrich, 2002. 113-130.

Rosenthal, Gabriele. Interpretative Sozialforschung: Eine Einführung. Weinheim, München: Juventa, 2005.

Rosenthal, Gabriele; Köttig, Michaela; Witte, Nicole; Blezinger, Anne. Biographisch-narrative Gespräche mit Jugendlichen. Chancen für das Selbst- und Fremdverstehen. Opladen: Barbara Budrich, 2006.

Stauber Barbara; Kaschuba, Gerrit. «Dem Verhältnis von Medienkompetenz und Gender-Kompetenz auf der Spur - Anregungen aus einer Evaluation medienpädagogischer Projekte.» Gender medienkompetent. Medienbildung in einer heterogenen Gesellschaft. Hrsg. v. Annette Treibel, Maja S. Maier, Sven Kommer u. Manuela Welzel. Wiesbaden: Verlag für Sozialwissenschaften, 2006. 327-341. 